\title{
Avaliação de Risco de Gestantes no Acesso à Unidades Básicas de Saúde
}

\section{Alternative Title: Risk Assessment of Pregnant Women Access to Basic Healthcare Units}

\author{
Edmir Parada Vasques Prado \\ Universidade de São Paulo - EACH \\ Av. Arlindo Bettio, 1000 - Ermelino Matarazzo \\ CEP: 03828-000 São Paulo/SP \\ eprado@usp.br
}

\author{
Eunice Almeida da Silva \\ Universidade de São Paulo - EACH \\ Av. Arlindo Bettio, 1000 - Ermelino Matarazzo \\ CEP: 03828-000 São Paulo/SP \\ eunice.almeida@usp.br
}

\begin{abstract}
RESUMO
A acessibilidade é uma condição essencial para o pleno exercício da cidadania e um direito cívico que não deve ser negado a nenhum cidadão. Apesar disso, muitas são as barreiras arquitetônicas encontradas no meio urbano, como calçadas quebradas e estreitas, pisos escorregadios, escadas e rampas excessivamente íngremes, entre outros. Pessoas com dificuldades de locomoção, sejam elas permanentes ou temporárias, são o público que mais sofrem com a dificuldade de acesso a espaços planejados, em especial aos serviços públicos de saúde, essenciais para a o bem-estar da população. Nesse contexto, o presente trabalho apresenta um sistema de avaliação de risco no acesso físico de gestantes a Unidades Básicas de Saúde (UBS). O sistema foi aplicado em duas unidades básicas de saúde e foi constatado que $35 \%$ das gestantes estão expostas a um risco médio e alto de complicações de saúde em função de obstáculos no trajeto às unidades de saúde.
\end{abstract}

\section{PALAVRAS-CHAVE}

Saúde Coletiva, Tecnologia da Informação, Análise de Risco.

\begin{abstract}
Accessibility is an essential condition for the full exercise of citizenship and a civic right that should not be denied to any citizen. Nevertheless, there are many architectural barriers found in urban areas, such as broken and narrow sidewalks, slippery surfaces, stairs and ramps excessively steep, among others. People with locomotion difficulties, whether permanent or temporary, are the public who suffer most from lack of access to planned areas, especially to public healthcare services, essential to the well-being of the population. In this context, this paper implemented a system to assess the risk of pregnant women to access Basic Health Units (UBS). The system was applied in two basic health units and we found that $35 \%$ of pregnant women are exposed to high or medium risk of health complications due to obstacles on the path to healthcare units..
\end{abstract}

\section{Categories and Subject Descriptors}

\footnotetext{
Permission to make digital or hard copies of all or part of this work for personal or classroom use is granted without fee provided that copies are not made or distributed for profit or commercial advantage and that copies bear this notice and the full citation on the first page. To copy otherwise, or republish, to post on servers or to redistribute to lists, requires prior specific permission and/or a fee.

SBSI 2016, May 17-20, 2015, Florianópolis, Santa Catarina, Brazil. Copyright SBC 2016.
}

J.3 [Computer Applications]: Life and Medical Sciences biology and genetics, health, medical information systems.

\section{General Terms}

Management, Measurement, Human Factors.

\section{Keywords}

Collective Health, Information Technology, Risk Analysis

\section{INTRODUÇÃ̃}

Em meados da década de 1970, no Brasil, foram implantados serviços que possibilitaram a descentralização do sistema de saúde. Esses serviços foram frutos das reivindicações por parte dos setores organizados da sociedade, que acabaram por gerar ações visando a melhoria do atendimento à saúde da população. Uma dessas ações foi a $8^{a}$ Conferência Nacional de Saúde, realizada em 1986, na qual foi apresentado o projeto da Reforma Sanitária Brasileira e a proposta de implantação de um Sistema Único de Saúde (SUS), garantida na Constituição de 1988.

O SUS foi planejado como uma organização dos serviços de saúde, pautada pelos princípios da universalidade (a assistência para todos), da integralidade (o atendimento ao indivíduo de maneira não fragmentada), da equidade (o direito a saúde por todos) e da acessibilidade (o acesso à assistência à saúde por todos os cidadãos) [1].

A Unidade Básica de Saúde (UBS) é considerada como a porta de entrada de todo cidadão ao SUS, pois se constitui no primeiro nível de atenção à saúde, designado como nível primário. As UBS são importantes no atendimento básico à saúde da população e das gestantes, no particular, pois nessas unidades é realizado o acompanhamento pré-natal.

O Programa de Humanização no Pré-natal de Nascimento do Ministério da Saúde Brasil (PHPN) tem como objetivo principal assegurar a melhoria do acesso, da cobertura e da qualidade do acompanhamento pré-natal, da assistência ao parto e puerpério às gestantes e ao recém-nascido, na perspectiva dos direitos de cidadania [2]. O PHPN preconiza que a gestante realize no mínimo seis consultas de acompanhamento pré-natal: uma no primeiro trimestre, duas no segundo trimestre e três no terceiro trimestre da gestação [2]. Apesar das políticas públicas de saúde, como o PHPN, reconhecerem a importância da acessibilidade das gestantes ao atendimento à saúde, o deslocamento delas até as UBS para realizar o acompanhamento pré-natal pode apresentar riscos à gravidez. Esses riscos são maiores ou menores dependendo das condições de 
acessibilidade no trajeto até a UBS e das condições clínicas que a gestante apresente.

Além disso, a questão da acessibilidade é uma reivindicação antiga, fortemente relacionada ao exercício da cidadania, como o direito de ir e vir. Assim, garantir cidadania é também eliminar barreiras urbanas, arquitetônicas e de transporte ao acesso de serviços e espaços de interesse de qualquer cidadão, em especial aos serviços de saúde, essenciais para a o bem-estar do grupo social. Dessa forma, esta pesquisa busca responder a seguinte pergunta de pesquisa: qual o nível de risco que as gestantes estão expostas no trajeto de casa até as unidades básicas de saúde?

Considerando este contexto, este trabalho tem como objetivo implementar um sistema que avalie o risco da acessibilidade das gestantes a partir de informações sobre: (1) a gestante e suas consultas de acompanhamento descritas no prontuário da paciente; (2) o trajeto efetuado pela gestante até a UBS; (3) literatura a respeito de enfermidades e suas relações com o risco gestacional; e (4) literatura sobre análise de risco.

A partir do objetivo geral foram definidos três objetivos específicos: (1) elaborar um modelo de avaliação de risco de acessibilidade de gestantes a partir de uma revisão da literatura; (2) elaborar uma especificação do software a partir do modelo de avaliação de risco; e (3) implementar e testar o software desenvolvido.

\section{FUNDAMENTAÇÃO TEÓRICA}

Nesta seção se encontra a fundamentação teórica usada para desenvolvimento de um sistema informatizado de análise de risco na acessibilidade de gestantes. Essa base teórica está dividida em três tópicos: Acessibilidade e Mudanças Biométricas na Gestante; Conceito de Risco; e Análise de Risco.

\subsection{Acessibilidade e Mudanças Biométricas na Gestante}

Todas as pessoas, entre as quais se incluem as que possuem algum tipo de deficiência ou as que temporariamente estão com a mobilidade reduzida, como as gestantes, têm direito ao acesso à educação, à saúde, ao lazer e ao trabalho. Essas áreas contribuem para a inserção social, desenvolvimento de uma vida saudável e de uma sociedade inclusiva [3].

As gestantes passam por mudanças biomecânicas e hormonais consideráveis, que geram recomendações médicas relacionadas à acessibilidade adequada e segura, como prevenção contra quedas e lesões. Vários estudos $[4,5,6,7,8]$ mostram que durante a gravidez ocorrem mudanças hormonais e biomecânicas que propiciam o relaxamento crescente dos ligamentos, além de um amolecimento cartilaginoso e aumento no volume de líquido sinovial e no espaço articular, o que contribui para a mobilidade articular aumentada e articulações mais instáveis, predispondo as gestantes às lesões.

Além disso, há o constante crescimento do útero, pelo aumento do peso do feto, o que leva ao aumento no peso e no tamanho das mamas da gestante. Esses são fatores que favorecem o deslocamento do centro de gravidade da mulher para cima e para frente, podendo acentuar a lordose lombar [9]. O aumento da carga e o desequilíbrio no sistema articular devido ao aumento da massa corpórea e de suas dimensões podem provocar perturbação do centro de gravidade e maior oscilação do centro de força, que levam a um equilíbrio instável e influenciam na biomecânica da postura.
Cabe também destacar a existência de fatores prévios como, por exemplo, a presença da osteoporose, que representa um fator de agravo ao risco de ocorrências de lesões.

\subsection{Conceito de Risco}

$\mathrm{O}$ conceito de risco é definido por diversos pesquisadores em diferentes áreas de atuação [10, 11, 12, 13, 14]. Alguns pesquisadores consideram o risco como um evento com efeito negativo ou positivo. Ou seja, riscos negativos representam ameaças a serem evitadas e riscos positivos oportunidades a serem aproveitadas. Entretanto, para efeito desta pesquisa, adotou-se a definição de autores que consideram apenas o efeito negativo [25, 26]. Em sua maioria, estes autores definem risco como um evento de condição incerta que, se ocorrer, provocará um efeito negativo no objeto de estudo. Além disso, segundo Perminova, Gustafsson e Wikström [16], risco, ao contrário da incerteza, se refere a possibilidade de estabelecimento de probabilidades, ou seja, as decisões são tomadas sob condições de probabilidades conhecidas.

O objetivo do gerenciar riscos é diminuir a probabilidade e o impacto dos eventos adversos. Segundo Carvalho e Rabechini Jr [15] existem seis processos de gerenciamento de risco, dos quais três deles referem-se a análise de risco, que faz parte do objetivo desta pesquisa: (1) identificação de riscos; (2) análise qualitativa dos riscos; e (3) análise quantitativa dos riscos.

\subsection{Análise de Risco}

$\mathrm{Na}$ análise de risco primeiro se identificam os riscos, em seguida faz-se uma análise qualitativa e quantitativa na qual se determinam as probabilidades de cada evento, bem como seu impacto.

\subsubsection{Identificação de Riscos}

O processo de identificação dos riscos serve para determinar quais riscos podem afetar o objeto de estudo e documentar as características desses riscos. Além disso, deve-se utilizar uma abordagem metódica e planejada, pois se for conduzida em várias direções diferentes, alguns riscos podem não ser identificados.

Existem inúmeras técnicas para identificação de riscos. As técnicas mais comuns são Brainstorming, Brainwriting, entrevista com especialistas, exame de documentos, Técnica Delphi, diagrama de causa e efeito e diagrama de influência, entre outras [15]. Essas são as técnicas mais comuns, mas determinadas áreas de conhecimento podem apresentar técnicas específicas. $\mathrm{Na}$ área de desenvolvimento de software as técnicas típicas de identificação de risco são: modelos de desempenho, modelos de custo, análise de redes, análise estatística de decisões e análise de fatores de qualidade, tais como, confiabilidade, disponibilidade e segurança [17].

O processo de identificação e documentação de riscos pode ser resumido em três etapas:

(1) Identificação dos riscos: Aplicação de técnicas para compor uma lista de riscos que afetam o estudo. Para efeito de utilização, ficou definido que o sistema terá aberta para que o usuário entre com as barreiras que oferecem risco potencial a gestante, ficando a cargo do mesmo um entendimento sobre possíveis riscos.

(2) Descrição dos riscos: Hillson [18] argumenta que um bom processo de identificação de risco deve proporcionar uma descrição detalhada do risco. Assim, deve ser feita em um único texto contemplando: a causa raiz do risco, associada a uma probabilidade; e o efeito (impacto).

(3) Categorização do risco: A grupar os riscos por afinidade, o que auxiliará na identificação de riscos em situações futuras. Tal 
agrupamento é representado por uma Estrutura Analítica de Riscos [15].

\subsubsection{Análise Qualitativa de Riscos}

A análise qualitativa dos riscos visa priorizar os riscos identificados na etapa anterior. Essa priorização é feita com base na avaliação da exposição ao risco de cada possível evento. Trata-se de uma abordagem que avalia a exposição ao risco, ou seja, a composição do impacto do risco e da probabilidade de sua ocorrência. Segundo Carvalho e Rabechini Jr [15] a principal ferramenta para a análise qualitativa dos riscos é a matriz de probabilidade e impacto. A Figura 1 apresenta uma matriz de probabilidade e impacto com três níveis de exposição ao risco.

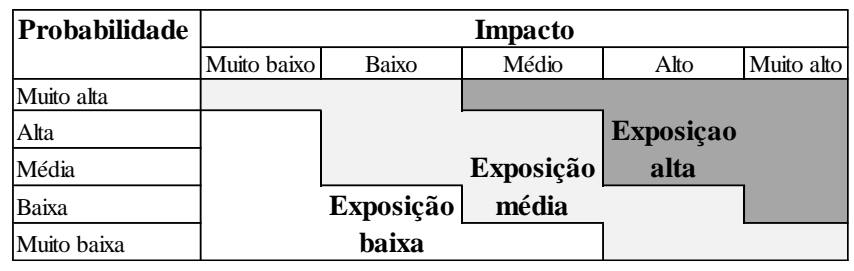

Figura 1. Matriz de probabilidade e impacto

Fonte: adaptado de Hillson (2000)

\subsubsection{Análise Quamtitativa de Riscos}

A análise quantitativa dos riscos analisa numericamente o efeito dos riscos identificados. O principal benefício desse processo é a produção de informações quantitativas para respaldar a tomada de decisões, a fim de reduzir o grau de incerteza que afeta o objeto de estudo. A análise quantitativa é executada nos riscos que foram priorizados na etapa de análise qualitativa, atribuindo uma classificação de prioridade numérica aos riscos.

Segundo Carvalho e Rabechini Jr. [15] a análise quantitativa pode contribuir com uma avaliação mais precisa, porém é menos utilizada nas organizações. Algumas técnicas utilizadas para análise quantitativa de riscos são: análise de sensibilidade, análise do valor monetário esperado, análise de impacto e esforço, árvore de decisão, modelagem e simulação.

Boehm [17] mostra um dos benefícios da análise quantitativa. Segundo o autor, eventos com alto impacto, ou alta probabilidade de ocorrência, podem ser classificados como prioritários erroneamente. Isto porque, o importante é a exposição ao risco, ou seja, o produto da probabilidade pelo impacto. A Figura 2 ilustra esta questão. As linhas do gráfico representam pontos de mesma exposição ao risco, na qual a linha A possui exposição ao risco maior que a linha $\mathrm{B}$, e esta maior que a linha C. Os pontos demarcados na Figura 2 também ilustram a importância de se considerar a exposição ao risco: entre os pontos $\mathrm{p}_{1}, \mathrm{p}_{2}$ e $\mathrm{p}_{3}$, o. ponto $\mathrm{p}_{1}$ é o que possui maior probabilidade de ocorrência e o ponto $\mathrm{p}_{3}$ é o de maior impacto. Entretanto, é o ponto p 2 o de maior exposição ao risco (produto da probabilidade pelo impacto), ou seja, aquele que deve ser considerado primeiro no gerenciamento da redução de risco.

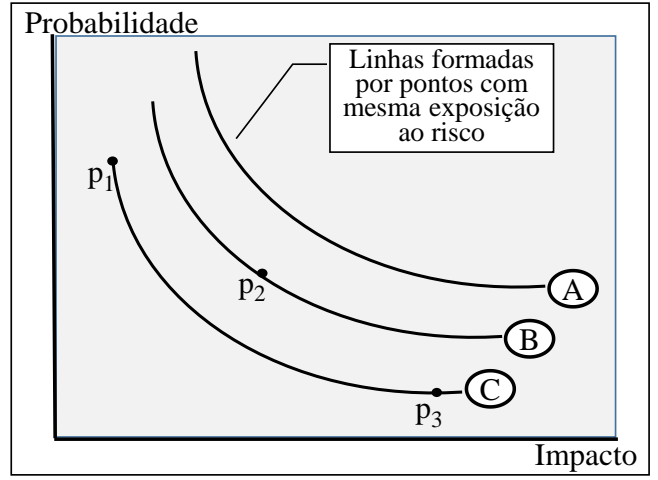

Figura 2. Exposição ao risco

Fonte: baseado em Boehm (1991)

\section{MÉTODO DE PESQUISA}

Esta seção apresenta os procedimentos metodológicos que foram aplicados à pesquisa. O primeiro item classifica o tipo de pesquisa, e os demais itens descrevem o modelo de pesquisa, as unidades de análise e as fases da pesquisa.

\subsection{Tipo de Pesquisa}

O tipo de pesquisa realizado neste estudo se insere dentro do paradigma da ciência de design (design science research). Este paradigma busca estender as fronteiras da capacidade humana e organizacional pela criação de novos e inovadores artefatos. Ele reside na confluência entre pessoas, organizações e tecnologia. As pesquisas desenvolvidas sob esse paradigma criam inovações que definem ideias, práticas e produtos por meio do qual a análise, implementação e gestão de sistemas de informação (SI) podem ser eficaz e eficientemente realizadas [19]. Neste paradigma, o conhecimento e o entendimento do domínio de um problema e sua solução são alcançados pela construção e aplicação do artefato projetado. É fundamentalmente um paradigma voltado para a solução de problemas [20].

Para March e Smith [21], os artefatos definidos no campo de SI, tais como, construtos, modelos, métodos, protótipos e sistemas implementados, permitem aos pesquisadores e praticantes compreender e endereçar os problemas inerentes a implantação de SI.

\subsection{Modelo para Avaliação de Risco de Acessibilidade de Gestantes}

O modelo desta pesquisa foi elaborado a partir da revisão da literatura e serviu como base para o desenvolvimento do software de avaliação de risco da acessibilidade de gestantes. O modelo considerou, além das condições do trajeto efetuado pela gestante, outros fatores que ampliam o grau de risco e do impacto que pode ser causado por uma ocorrência indesejada no trajeto até a UBS, como por exemplo uma queda devido a um buraco, a um piso escorregadio, entre outros. Entre esses fatores destacam-se enfermidades que pode agravar a ocorrência de um evento negativo durante o trajeto. Como exemplo, a osteoporose, que poderia se tornar em um fator de ampliação do impacto causado pela queda ocorrida em um buraco mal sinalizado ou mal protegido na via pública. Outros fatores como o tempo de gestação, histórico familiar, entre outros, podem também contribuir para tornar o evento mais danoso à gestante.

O modelo para avaliação de risco de acessibilidade de gestantes está ilustrado na Figura 3. A apresentação dos conceitos que 
compõem o modelo, bem como os critérios de mensuração, estão descritas a seguir.

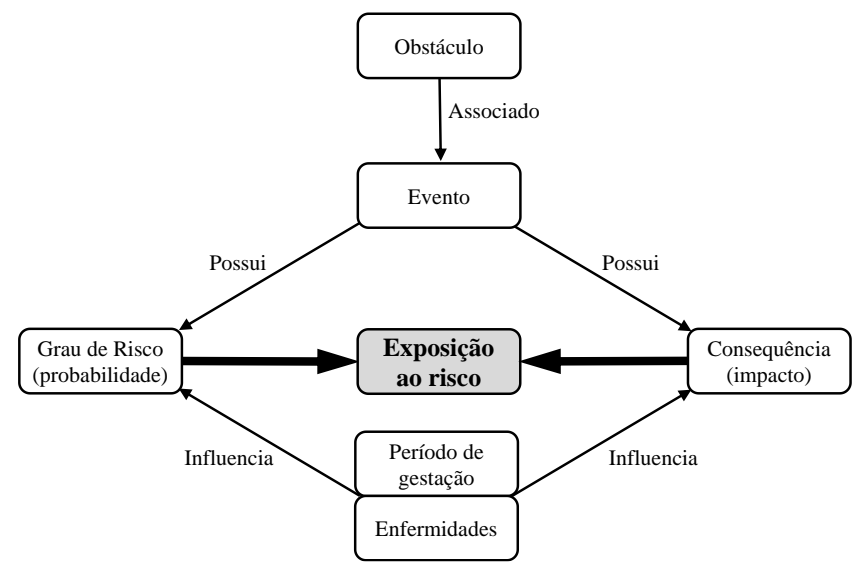

Figura 3. Modelo para avaliação de risco

Fonte: próprio autor

(1) Obstáculo. Toda e qualquer barreira à acessibilidade da gestante durante o trajeto do domicílio à UBS. Os obstáculos foram classificados de acordo com o grau de severidade em relação à acessibilidade, em três categorias: baixa (B), média (M) e alta (A) intensidade. Exemplos de obstáculos são: buracos, escadas, calçadas em mau estado de conservação, entre outros.

(2) Evento. Toda ocorrência que pode ser originada por um obstáculo e que gera consequências à saúde da gestante. A partir de um obstáculo, como um buraco, pode se ter um evento de fratura, ou um evento de rompimento de ligamento, entre outros.

(3) Grau de risco. Refere-se a probabilidade da ocorrência de um determinado evento associado à um obstáculo no trajeto da gestante à UBS. O grau de risco foi classificado em cinco níveis: muito baixo $(\mathrm{MB})$, baixo $(\mathrm{B})$, médio $(\mathrm{M})$, alto $(\mathrm{A})$ e muito alto (MA). O período gestacional influencia o grau de risco. A mensuração do grau de risco de um determinado evento é dada pela fórmula:

$$
\mathrm{G}_{\mathrm{E}}=\mathrm{P} * \mathrm{~S}
$$

Onde $\mathrm{G}_{\mathrm{E}}=$ grau de risco de um evento; $\mathrm{P}$ = período gestacional; e S severidade do obstáculo.

(4) Impacto. É a consequência gerada por um evento. O impacto foi classificado em cinco níveis: muito baixo (MB), baixo (B), médio (M), alto (A) e muito alto (MA). O período gestacional e as enfermidades associadas à gestante influenciam no impacto gerado por um evento. Alguns exemplos são: os efeitos de uma queda são amplificados quando a gestante tem problemas de osteoporose; longas caminhadas em dias de alta temperatura podem ter consequências em gestantes com problemas de pressão baixa, entre outras. A mensuração do impacto de um determinado evento é dada pela fórmula:

$$
\mathrm{I}_{\mathrm{E}}=\mathrm{P} * \mathrm{E}_{\mathrm{E}}
$$

Onde $\mathrm{I}_{\mathrm{E}}=$ impacto causado por um evento; e $\mathrm{E}_{\mathrm{E}}=$ enfermidade da gestante associada ao evento.

(5) Exposição ao risco. Refere-se à intensidade de exposição da gestante aos riscos associados a dificuldades de acesso à UBS.
A exposição ao risco é calculada pelo produto do grau de risco pelo seu impacto, com ilustra a fórmula:

$$
\mathrm{ER}_{\mathrm{E}}=\mathrm{G}_{\mathrm{E}} * \mathrm{I}_{\mathrm{E}}
$$

Onde $\mathrm{ER}_{\mathrm{E}}=$ exposição ao risco pelo evento.

\subsection{Unidades de Análise}

Foram selecionadas duas UBS na zona leste de São Paulo no bairro de Ermelindo Matarazzo. Trata-se de UBS que atende gestantes que pertencem a famílias de baixa renda ou que apresentam problemas de locomoção e acessibilidade às UBS.

Os problemas de acessibilidade dessas UBS são corroborados pelo Boletim Técnico da Escola Politécnica da Universidade de São Paulo [22], que mostra que a zona Leste, principalmente, a região de São Miguel Paulista e Ermelindo Matarazzo, teve uma expansão urbana desordenada. Isso resultou em déficits de infraestrutura que estão concentrados em iluminação pública e pavimentação. Podese observar que há trajetos na região Leste de São Paulo que apresentam riscos para a gestante, devido às condições de pavimentação das calçadas, presença de buracos, escadas, rampas, dentre outros, não projetados sob padrões exigidos pela lei que os regularizam.

Como consequência, as UBS selecionadas representam unidades de análise de interesse na aplicação do modelo para avaliação de risco de acessibilidade de gestantes, objeto deste trabalho.

\subsection{Fases da Pesquisa}

Esta pesquisa foi desenvolvida em três fases. A primeira fase constituiu o levantamento bibliográfico apresentado na Seção 2. Esse levantamento considerou os tópicos de análise de risco e de saúde da gestante.

A segunda fase, definiu um modelo para avaliação de risco na acessibilidade de gestantes. A construção desse modelo partiu dos conceitos apresentados na revisão bibliográfica e propôs uma alternativa para avaliação da exposição ao risco a partir da probabilidade de ocorrência e do impacto gerado por obstáculos no trajeto executado pela gestante até a UBS.

$\mathrm{Na}$ terceira fase, realizou a implementação do modelo proposto. Para isso realizaram-se seis etapas: (1) levantamento de requisitos do sistema; (2) especificação do projeto lógico do sistema; (3) especificação do projeto físico do sistema; (4) implementação do software; (5) teste e validação; e (6) documentação.

Por último, na quarta fase, aplicou-se o software desenvolvido em duas UBS da zona leste de São Paulo, cujos resultados obtidos estão descritos nas seções de análises e discussões de resultados.

\section{DESCRIÇÃO DO PROJETO DE IMPLEMENTAÇÃO DO MODELO}

O projeto de desenvolvimento do software para avaliação de risco de acessibilidade de gestantes contou com uma equipe de sete pessoas, sendo dois professores e cinco alunos de graduação com as seguintes responsabilidades.

(1) Professor do curso de Sistemas de Informação (SI). Responsável pelo desenvolvimento do modelo de análise de risco e do software que implementou o modelo.

(2) Professora do curso de Obstetrícia (OBS). Responsável pela articulação entre a área de Sistemas de Informação e a área de Obstetrícia; responsável pela coleta de dados nas Unidades Básicas de Saúde. 
(3) Alunos de graduação. Contou com um aluno de graduação do curso de SI e nove alunos do curso de OBS. O primeiro atuou nas atividades de desenvolvimento do software e os demais nas atividades de coleta de dados junto as UBS.

As características principais do projeto estão descritas a seguir.

\subsection{Levantamento de Requisitos e Especificação do Projeto}

Os requisitos foram levantados por meio de reuniões realizadas com a equipe do projeto. Os principais requisitos definidos para o sistema foram:

(1) Interface do sistema com o prontuário das gestantes. Tem como objetivo associar o risco avaliado com características da gestante.

(2) Rotina de avaliação de risco deve possuir relatório que apresente resultados intermediários e que seja possível analisar a exposição de risco calculada pelo sistema. Tem como finalidade permitir analisar se as regras de avaliação de risco do sistema representam a especificação do modelo.

(3) Interface do sistema. Apesar do sistema ter apenas a finalidade de testar o modelo nas UBS, a interface do sistema deve permitir que profissionais de saúde possam proceder as avaliações de risco de forma rápida e fácil, sem necessidade de conhecimento sobre software e hardware.

\subsection{Tecnologias e Ferramentas Utilizadas}

O sistema foi modelo com oito entidades conforme ilustra a Figura 4. Essas entidades representam:

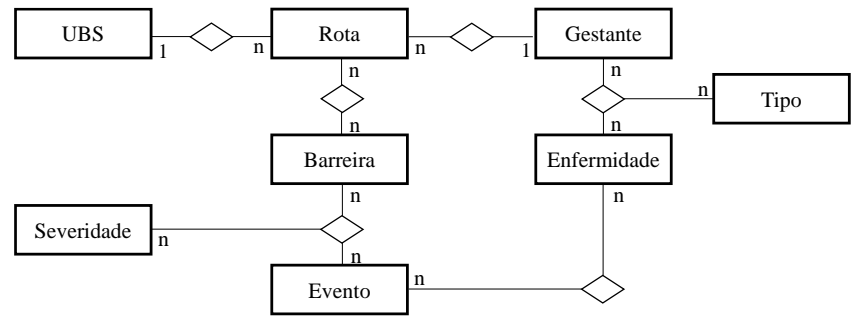

Figura 4. Modelo simplificado de entidade e relacionamento Fonte: próprio autor

(1) UBS. Unidades básicas de saúde analisadas pelo sistema.

(2) Gestante. Informações gerais da gestante presente no prontuário dela.

(3) Rota. Representa o trajeto das gestantes do local onde moram até a UBS.

(4) Barreira. Todo obstáculo que possa ser apresentado para a gestante no percurso até a UBS.

(5) Evento. Qualquer ocorrência com consequências à saúde da gestante provocado por uma barreira apresentada no trajeto.

(6) Severidade. Grau de dano que o evento pode causar à saúde da gestante.

(7) Enfermidade. Doenças pré-existentes ou não que possa estar no histórico da gestante e agravar ainda mais o dano causado pelo evento.

(8) Tipo (de enfermidade). Relação de enfermidades presentes no prontuário da gestante que podem influir na ocorrência do evento.
O software foi implementado com uso de ferramentas, em sua maioria, disponíveis gratuitamente (opensource). Entre essas ferramentas, cinco se destacam: (1) Apache HTTP Server, serviço HTTP para acesso web, que cria uma camada de interface para a aplicação e o acesso à internet; (2) Python, foi a linguagem de programação para desenvolvimento de alto nível, interpretada e funcional; (3) Django, framework de desenvolvimento web para Python; (4) Bootstrap, framework HTML para desenvolvimento de sites; e (5) PostgreSQL, como banco de dados relacional.

Para disponibilizar o sistema na internet, foi necessário criar um ambiente de aplicação web. Para tal, foi utilizado um serviço temporário gratuito oferecido pela Amazon Web Services constituído de: (1) Amazon EC2, serviço para ambiente de aplicação que cria máquinas que podem ser configuradas; e Amazon RDS, que é um serviço para uso de banco de dados. Adicionalmente, no serviço de versionamento, foi utilizada a plataforma BitBucket (bitbucket.org) que armazena todo o código fonte e gere a comunicação de acesso ao código.

\section{APLICAÇÃO DO MODELO NAS UBS}

O modelo referido foi aplicado na Unidade Básica de Saúde de Ermelino Matarazzo e Jardim Keralux. A UBS Ermelino Matarazzo está localizada no extremo da zona Leste da cidade de São Paulo; é administrada pela Organização Social de Saúde (OSS), SECONCI, em parceria com a Secretaria Municipal de Saúde. No mês de outubro/2015 contava com um cadastro de 130 gestantes.

A UBS Jardim Keralux está localizada no extremo da zona Leste da cidade de São Paulo; é administrada pela Secretaria Municipal de Saúde da cidade de São Paulo. No mês de outubro/2015 contava com um cadastro de 93 gestantes.

As duas UBS investigadas têm uma média de $30 \%$ de faltas às consultas para acompanhamento do pré-natal. As faltas estão relacionadas a diversos fatores, dentre eles a dificuldade de acesso que as gestantes referem para caminhar até a UBS.

Os resultados da aplicação do modelo de avaliação do risco de acessibilidade de gestantes estão apresentados em três seções subseções: Estatísticas sobre as Gestantes; Resultados da Aplicação do Modelo; e Análise e Discussão.

\subsection{Dados sobre as Gestantes}

Os dados das gestantes estão apresentados na Tabela 1. A maioria das gestantes estão na faixa etária de 16 a 30 anos (78\%), sendo apenas $4 \%$ acima de 40 anos, ou seja, em idade que exige maior atenção. A distribuição das gestantes pelas faixas etárias é bem semelhante entre as três raças (branca, negra e parda). No caso do estado civil houve diferença, ou seja, as gestantes negras com estado civil casada são de frequência maior que as demais. Entretanto, se considerarmos as que convivem com companheiro essa diferença diminui.

Em relação aos dados da situação econômica da família, houve diferença significativa, apesar de não haver diferença no grau de escolaridade. Ou seja, o grau de escolaridade é semelhante entre as diversas raças, predominando a formação em ensino fundamental completo com $65 \%$ da amostra. Apesar da similaridade na escolaridade, há diferença na situação econômica da família. A maioria das gestantes estão nas faixas de renda mais baixa (classe E e D), o que era de se esperar pela escolha de UBS em regiões carentes da zona leste de São Paulo. Cabe destacar ainda que as gestantes da raça branca possuem situação econômica pouco melhor que as da raça negra e parda. Essa diferença tem 
significância estatística ao nível de 5\%, aplicando-se o Teste Exato de Fischer [27]. 14\% das gestantes da raça branca pertencem a classe social $\mathrm{C}$, e nenhuma das gestantes das demais raças pertencem a esta classe social.

Tabela 1. Dados sobre as Gestantes

\begin{tabular}{|c|c|c|c|c|c|c|c|c|}
\hline \multirow[t]{2}{*}{ Raça } & Idade & $\begin{array}{l}\text { Escola- } \\
\text { ridade }\end{array}$ & \multicolumn{2}{|l|}{$\begin{array}{c}\text { Estado } \\
\text { Civil }\end{array}$} & \multicolumn{2}{|c|}{$\begin{array}{l}\text { Situação } \\
\text { Familiar }\end{array}$} & \multicolumn{2}{|c|}{$\begin{array}{c}\text { Renda } \\
\text { Familiar }\end{array}$} \\
\hline & Nível \% & Nível \% & Faixa & $\%$ & Faixa & $\%$ & Nível & $\%$ \\
\hline \multirow[t]{2}{*}{ Branca } & $16-20 \quad 29$ & $0-3$ & Casada & 57 & $I^{(*)}$ & 86 & $\mathrm{E}^{(* *)}$ & \\
\hline & $21-30 \quad 43$ & $4-7$ & Solteira & 43 & II & 14 & D & \\
\hline \multirow[t]{2}{*}{$43 \%$} & $31-40 \quad 14$ & $8-11$ & & & III & 0 & $\mathrm{C}$ & \\
\hline & $>40$ & $>12$ & & & IV & 0 & B & \\
\hline \multirow[t]{2}{*}{ Negra } & $16-20 \quad 40$ & $0-3$ & Casa & 80 & & 80 & $E$ & \\
\hline & $21-30 \quad 40$ & $4-7$ & Solteira & 20 & II & 0 & D & \\
\hline \multirow[t]{2}{*}{$22 \%$} & $31-40 \quad 20$ & $8-11$ & & & III & 0 & $\mathrm{C}$ & \\
\hline & $>40$ & $>12$ & & & IV & 20 & B & \\
\hline \multirow[t]{2}{*}{ Parda } & $16-20 \quad 50$ & $0-3$ & Casada & 55 & I & 64 & $E$ & \\
\hline & $21-30 \quad 30$ & $4-7$ & Solteira & 46 & II & 27 & D & \\
\hline \multirow[t]{2}{*}{$35 \%$} & $31-40 \quad 20$ & $8-11$ & & & III & 0 & $\mathrm{C}$ & \\
\hline & $>40 \quad 0$ & $>12$ & & & IV & 9 & $\mathrm{~B}$ & \\
\hline \multirow[t]{2}{*}{ Total } & $16-20 \quad 41$ & $0-3$ & Casada & 61 & $\mathrm{I}$ & 74 & $E$ & \\
\hline & $21-30 \quad 37$ & $4-7$ & Solteira & 39 & II & 17 & D & \\
\hline \multirow[t]{2}{*}{$100 \%$} & $31-40 \quad 18$ & 8-11 & & & III & 0 & $\mathrm{C}$ & \\
\hline & $>40 \quad 4$ & $>12$ & & & IV & 9 & B & \\
\hline
\end{tabular}

Legenda: (*) I - Convive com companheiro II - Convive com familiares III - Vive só IV - Vive com filhos

(**) Lista de classes sociais IBGE

Pelos dados apresentados observa-se que a amostra utilizada para aplicação do modelo de avaliação de risco de acessibilidade é adequada, pois tratam-se de gestantes com baixo nível de escolaridade e renda, vivendo em locais de periferia com dificuldade de assistência à saúde e de acesso as UBS. O trajeto executado por essas gestantes no deslocamento até a UBS apresenta dificuldades que se mostram interessantes de serem avaliadas pelo sistema. Por fim, cabe destacar ainda que o período gestacional médio das gestantes da amostra é de 24 semanas.

\subsection{Resultados da Aplicação do Modelo}

O sistema de avaliação de risco de acessibilidade foi aplicado na amostra de gestantes obtidas nas duas UBS. O trajeto feito pelas gestantes foi acompanhado pelo grupo de pesquisa. As barreiras e obstáculos que mais se destacaram foram os caminhos estreitos das calçadas e o estado do piso, como mostra a Tabela 2. Cabe destacar ainda que $95,7 \%$ das gestantes se deslocam a pé até a UBS e 4,3\% usam o trem. $68 \%$ dos trajetos feitos pelas gestantes são em calcadas estreitas e com piso de péssima qualidade, ou seja, com buracos, irregularidades, falta de calçamento entre outros problemas.

Além de se deslocar até a UBS para atendimento médico, 52,2\% das gestantes tem se deslocar para o trabalho ou para buscar o filho na escola. Isso aumenta o risco no deslocamento das gestantes para essas finalidades, pois a maioria desses deslocamentos é feito a pé, por trajeto se qualidade semelhante ao caminho feito até a UBS.

Conforme o modelo concebido para a avaliação do risco de acessibilidade, as enfermidades amplificam os danos causados por possíveis ocorrências, tais como, queda em buracos, esforço excessivo em ladeiras, entre outros. Por outro lado, a frequência de enfermidades pré-existentes ou adquiridas pelas gestantes da amostra foi baixa (22\%). Sendo que a hipertensão é a que mais se destaca, presente em $9 \%$ das gestantes.
Tabela 2. Avaliação do Risco de Acessibilidade

\begin{tabular}{|c|c|c|c|c|c|c|c|}
\hline \multirow[t]{3}{*}{ Raça } & \multicolumn{3}{|c|}{ Barreiras } & \multirow{2}{*}{\multicolumn{2}{|c|}{ Enfermidades }} & \multirow{2}{*}{\multicolumn{2}{|c|}{ Risco }} \\
\hline & Largura & \multicolumn{2}{|l|}{ Piso } & & & & \\
\hline & Nível \% & Nível & $\%$ & Nível & $\%$ & Nível & $\%$ \\
\hline \multirow[t]{2}{*}{ Branca } & Péssimo 57 & Péssimo & 57 & Hipertensão & 0 & Alto & 0 \\
\hline & Ruim & Ruim & 29 & Diabetes & 0 & Médio & \\
\hline \multirow[t]{2}{*}{$43 \%$} & Regular & Regular & 14 & Out & 0 & Baixo & 100 \\
\hline & Bom & Bom & 0 & Nenhuma & 100 & & \\
\hline \multirow[t]{2}{*}{ Negra } & Péssimo & Péssimo & 80 & Hiperten & 20 & Alto & 25 \\
\hline & Ruim & Ruim & 20 & Diab & 0 & Médio & \\
\hline \multirow[t]{2}{*}{$22 \%$} & Regular & Regular & 0 & Outras & 0 & Baixo & 50 \\
\hline & Bom & Bom & 0 & Nenhuma & 80 & & \\
\hline \multirow[t]{2}{*}{ Parda } & Péssimo 70 & Péssimo & 70 & Hipertensão & 9 & Alto & 0 \\
\hline & Ruim & Ruim & 30 & Diab & 0 & Médio & \\
\hline \multirow[t]{2}{*}{$35 \%$} & Regular & Regular & 0 & Outras & 27 & Baixo & 44 \\
\hline & Bom & Bom & 0 & Nenhuma & 64 & & \\
\hline \multirow[t]{2}{*}{ Total } & Péssimo & Péssimo & 68 & Hipertensão & 9 & Alto & 5 \\
\hline & Ruim & Ruim & 27 & Diab & 0 & Médio & \\
\hline \multirow[t]{2}{*}{$100 \%$} & Regular & Regular & 5 & Outr & 13 & Baixo & 65 \\
\hline & Bom & Bom & 0 & Nenhuma & 78 & & \\
\hline
\end{tabular}

De um modo geral, pode-se dizer que a hipertensão em gestantes pode evoluir para doenças como a hipertensão crônica ou gestacional, pré-eclâmpsia e eclampsia; doenças caracterizadas por alguns sinais clínicos e sintomas como, tonturas, mal-estar generalizado, convulsões, dentre outros [23]. Considerando esses sintomas somados as alterações biométricas, o modo de andar e o equilíbrio são afetados, podem amplificar os riscos de quedas e traumas relacionados a dificuldades na acessibilidade da gestante a UBS. Dependendo da gravidade da queda pode haver comprometimento no desenvolvimento do feto e na própria vida da gestante. Além disso, no Brasil a hipertensão representa a primeira causa de morte materna (37\%), sendo que a maior proporção se concentra nas regiões Norte e Nordeste do país [24].

A aplicação do modelo de avaliação de risco à situação das gestantes mostrou que $30 \%$ delas tem um risco médio de ter problemas de saúde como consequência da falta de acessibilidade às UBS. Ou seja, requer muita atenção da gestante no trajeto a UBS para evitar possíveis problemas. Mais ainda, $5 \%$ delas tem um risco alto de acessibilidade às UBS. Ou seja, recomenda-se que evitem o trajeto, buscando outras alternativas de locomoção. Uma alternativa aos problemas descritos seria a atuação do programa Estratégia da Saúde da Família (ESF), disponível em algumas UBS, para realizar as consultas no domicílio da paciente.

\subsection{Análise e Discussão}

O software implementado a partir do modelo de avaliação de risco de acessibilidade apresentou resultados positivos. $\mathrm{O}$ risco no trajeto até a UBS foi mensurado, permitindo fazer comparações entre as gestantes. Com base no risco de trajeto de cada gestante até a UBS foi possível identificar gestantes com maior risco de acessibilidade. Isso permite priorizar o atendimento as gestantes em casa. Esse tipo de atendimento já existe nas UBS, mas o critério de priorização não considera a dificuldade e nem o risco associado ao trajeto. Dessa forma, o software implementado pode contribuir com o melhor gerenciamento das gestantes por parte das UBS.

O risco de acessibilidade avaliado pelo software precisa ser considerado dentro dos diversos riscos presentes durante o período gestacional. Há outros riscos, alguns até de maior relevância, que não fazem parte do escopo deste trabalho, mas que devem ser considerados pelas UBS. A implementação deste software e sua aplicação a uma amostra de gestantes de duas UBS contribuiu 
também para identificar esses outros riscos associados a gestante. Esses outros riscos podem ser classificados em riscos associados à saúde e riscos associados ao contexto social das gestantes.

Os outros riscos associados à saúde e identificados durante a aplicação do software foram principalmente as ausências às consultas médicas e o uso de fumo durante o período gestacional. $17,4 \%$ das gestantes fumam. Mais ainda, além de fumar $4,4 \%$ consomem álcool e drogas ilícitas. Essas ocorrências certamente implicam em riscos à gestante maiores do que os riscos de acessibilidade identificados.

Os riscos associados ao contexto social das gestantes, que foram identificados nesta pesquisa, se relacionam à influência da criminalidade e até mesmo da liderança comunitária. A criminalidade é o mais relevante. Ela interfere na frequência com que as gestantes comparecem às UBS e até mesmo na entrada de equipes de saúde para atendimento na comunidade. No caso da liderança comunitária, foi percebido que, às vezes, ela exerce um papel de priorização no atendimento de gestantes que é melhor desempenhado pelo software, evitando conflitos e interesses pessoais.

\section{CONSIDERAÇÕES FINAIS}

Este trabalho teve como objetivo implementar um sistema de avaliação de risco da acessibilidade das gestantes. Para isso foi elaborado um modelo de avaliação de risco a partir de uma revisão da literatura. $\mathrm{O}$ levantamento bibliográfico realizado mostrou que há uma carência de trabalhos, na área da saúde e de sistemas de informação, que aborde a questão da acessibilidade da gestante. $\mathrm{O}$ que a literatura tem discutido são aspectos étnicos e culturais, mas não tem explorado as condicionantes macro e micro estruturais urbanas, relativas à própria organização do território e dos meios de circulação de veículos e pessoas.

O presente trabalho trouxe uma contribuição importante e inovadora, uma vez que mostrou as dificuldades e riscos encontrados na trajetória das gestantes a UBS. Tais riscos podem contribuir com o Programa de Humanização no Pré-natal e Nascimento (PHPN), instituído pelo Ministério da Saúde em 2000, por meio do monitoramento da acessibilidade da gestante, auxiliando a elevar a qualidade do acompanhamento do pré-natal nas UBS.

Além disso, o presente trabalho trouxe para reflexão as questões prévias a entrada dos usuários nos serviços de saúde, suas ruas, calçadas, rampas, em especial às gestantes, grupo com mobilidade condicionada temporária. Entretanto, o modelo desenvolvido neste trabalho pode ser ampliado para a acessibilidade de todos os usuários de serviços de saúde.

Finalizando, concluiu-se que a aplicação dessa primeira versão do software permitiu obter uma compreensão mais profunda da realidade das gestantes e os riscos envolvidos na gravidez. Como trabalhos futuros, pretende-se ampliar a aplicação do modelo em outras UBS e incrementar novas funcionalidades. Assim, será possível ter uma ferramenta que mitigue os riscos de acessibilidade das gestantes a UBS.

\section{REFERÊNCIAS}

[1] Silva, E. A. 2012. Sociologia aplicada à enfermagem. Ed. Manole: São Paulo.

[2] BRASIL, Ministério da Saúde. 2002. Programa Humanização do Parto, Humanização do Pré-Natal e Nascimento. http://bvsms.saude.gov.br/bvs/publicacoes/parto.pdf. Acesso em 18/04/2015.

[3] Pagliuca, L. M. F., Aragão, A.E. A., Almeida, P. C. 2007. Acessibilidade e deficiência física: identificação de barreiras arquitetônicas em áreas internas de hospitais de Sobral, Ceará. Revista da Escola de Enfermagem da USP, 41, 4, 581-588.

[4] Hollingsworth, D.R. 1985. Maternal Metabolism in Normal Pregnancy and Pregnancy Complicated by Diabetes Mellitus. Clinical Obstetrics Gynecology, 28, 3, 457-472.

[5] Ireland M.L., and Ott S. M. 2000. The effects of pregnancy on the musculoskeletal system. Clinical Orthopaedics \& Related Research, 372, 169-179.

[6] Neme, B. 2005. Obstetrícia Básica, $3^{a}$ edição. São Paulo: Livraria Sarvier.

[7] Okuno, E., Fratin, L. 2003. Desvendando a Física do Corpo Humano - Biomecânica. Ed. Manole: São Paulo.

[8] Vullo V.J., Richardson J.K., and Hurvitz E.A. 1996. Hip, knee, and foot pain during pregnancy and the postpartum period. Journal of Family Practice, 43, 1, 63-68.

[9] Martins, R. F., and Silva, J. L. P. 2005. Prevalência de dores nas costas na gestação. Revista da Associação Médica Brasileira, 51, 3, 144-147.

[10] Barki, H., Rivardi, S., and Talbot, J. 1993. Toward an assessment of software development risk. Journal of Management Information Systems, 10, 2, 203-225.

[11] Keil, M., Cule, P. E., Lyytinen, K., and Schimidt, R. C. 1998. A framework for identifying software project risk. Communication of the ACM, November, 41, 1998.

[12] Jiang, J. J., and Klein, G. 1999. Risks to different aspects of system success. Information \& Management, 36, 263-272.

[13] Fransman, M. 2000. Evolution of the telecommunications industry: into the internet age. University of Edinburgh.

[14] Valeriano, D. 2005. Moderno Gerenciamento de Projetos. São Paulo: Pearson Prentice Hall.

[15] Carvalho, M. M., and Rabequni Jr., R. 2011. Fundamentos em gestão de projetos, $3^{a}$ edição. São Paulo: Atlas.

[16] Perminova, O., Gustafsson, M., and Wikström, K. 2008. Defining uncertainty in projects: a new perspective. International Journal of Project Management, 26, 73-79.

[17] Boehm, B. W. 1991. Software risk: management principles and practices. IEEE Software, 8, 1, 32-41.

[18] Hillson, D. 2001. Extending the risk process to manege opportunities. PMI Europe.

[19] Denning, P. J. 1997. A new social contract for research. Communications of the ACM, 40, 2, 132-134.

[20] Hevner, A. R., March, S. T., Park, J., and Ram S. 2004. Design Science in Information Systems Research. Manegement Information System Quarterly, 28, 1, 75-105.

[21] March, S. T., and Smith, G. 1997. Design and natural science research on information technology. Decision Support Systems, 15, 4, 251-266.

[22] Massara, V. M., and Zmitrowicz, W. 2003. Infraestrutura e uso do solo no município de São Paulo: o centro expandido e a região de São Miguel Paulista. São Paulo: EPUSP, 2003 
[23] Sibai B.M. 2002. Chronic hypertension in pregnancy. Obstetrics and Gynecology, 100, 369-77.

[24] Laurenti R., Jorge M.H.P.M., and Gotlieb S.L.D. 2004. A mortalidade materna nas capitais brasileiras. Revista Brasileria de Epidemiologia, 7, 4, 449-60.

[25] Schmidt, R., Lyytinen, K., Keil, M., Cule, P. 2001. Identifying software project risks: an international Delphi study. Journal of Management Information Systems. 17 (4), $5-36$.

[26] Liu, S., Wang, L., 2014a. Understanding the impact of risks on performance in internal and outsourced information technology projects: the role of strategic importance.
Internationl Journal of Project Management, 32 (8), 14941510 .

[27] Hair, Jr., J. F., Black, W. C., Babin, B. J., Anderson, R. E., and Tatham, R. L. 2006. Multivariate Data Analysis (6th ed.), Upper Saddle River, NJ: Pearson Prentice Hall. 